\title{
An Existence Theorem for General Control Problems of Nonlinear Evolution Equations of First Order
}

\author{
B. Krause
}

Für die Lösung optimaler Steuerprobleme mit dér Zustandsgleichung $\dot{y}+\nu A \dot{y}+B(\dot{y})=u$, die durch einen linearen Operator $A$ und einen nichtlinearen Operator $B$ mit $\|B(y)\|_{n} \leqq c$ $\times\left(1^{\prime}+\|y\|_{m}{ }^{r}\right)$ gekennzeichnet ist, wird ein Existenzsatz bewiesen.

Доказывается теорема существования решения для проблсм оптимального управления системами, которые характеризуются уравнением состояния $\dot{y}+v A y+B(y)={ }^{\prime} u$ с тинейым.оператором $A$ и нејинейым оператором $B$, где $\|B(y)\|_{n} \leqq c\left(1+\|y\|_{m}{ }^{\top}\right)$.

An existence theorem for the solution of optimal control problems of systems governed by the state equation $\dot{y}+v A y+B(y)=u$ is proved, where $A$ is a linear operator and $B$ a nonlinear operator with $\|\left. B(y)\right|_{n} \leqq c\left(1+\left.\right|_{i} y{ }^{\prime} \mid m^{r}\right)$.

\section{Introduction}

A new method of proving existence theorems for solutions of control problems for systenis governed by nonlinear evolution equations was proposed in [1]. There this method was applied to the Navier-Stokes system with positive or negative. viscosity and to the Euler system.. A similar approach was used in [5] to treat control problems for systems with the state equation $\dot{y}-\Delta y=y^{3}+u$ in a bounded domain with appropriate boundary and initial conditions. In both cases one can prove existence theorems for control problems without an assumption concerning the unique solvability of the state equation for the controlled system. One only needs that at least one admissible couple of state and control exists for which the state equation is fulfilled and the objective functional $J$ is finite. Since this assumption gives an additional a priori estimate for the solution of the state equation, the existence theorem to be proved for the considered problem depends in an essential way on parameters which describe the regularity properties of the functional $J$.

The aim of the present paper is the extension of existence theorems given in $[1,2,5]$ to a class of control problems for systems which are governed by evolution equations with polynomial nonlinearities. Using general interpolation inequalities one can also improve the estimates of the linear and nonlinear operator in the state equation (cf. Lemma 2:5 and Lemma 2.6): So it is possible to enlarge the region of parameter values for which the control problem has a solution in corresponding function spaces.

A general approach to proving existence theorems for extremal problems is developed in [3]. Similar existence theorems for control problems, which are proved in $[4,6]$, are based on the Weierstrass theorem and the theory of monotone operators. 


\section{Function spaces}

Let $H$ be a real Hilbert space with norm $\|\cdot\|$ and inner product $(\cdot, \cdot)$. Let $A: H \rightarrow H$ be a selfadjoint positive definite operator with discrete spectrum. We denote the eigenvalues of $A$ by $\lambda_{i}, 0<\lambda_{1} \leqq \lambda_{2} \leqq \ldots, \lambda_{i} \rightarrow \infty$ as $i \rightarrow \infty$, and the corresponding orthonormal basis in $H$ consisting of the eigenvectors of $A$ by $\left\{e_{i}\right\}_{i, 1}^{\infty}$. For each $s \in \mathbb{R}$ we set

and,

$$
H^{s}=\left\{v=\sum_{i=1}^{\infty} v_{i} e_{i}: v_{i} \in \mathbb{R}^{\prime},\|v\|_{s}=\left(\sum_{i=1}^{\infty} \lambda_{i}{ }^{8}\left|v_{i}\right|^{2}\right)^{1 / 2}<\infty\right\}
$$

$$
E_{\infty}=\left\{v=\sum_{i=1}^{i *} v_{i} e_{i}: v_{i} \in \mathbb{R} ; i^{*}<\infty\right\} .
$$

'The set $E_{\infty}$ is dense in $H^{s}$ for all $s \in \mathbb{R}$. From the definition of $H^{s}$ it follows that for any $s \in \mathbb{R}$ the operator $A: H^{s} \rightarrow H^{s-2}$ is.continuous and that for $s \not 0$ the norm $\|\cdot\|_{-s}$ is equal to the negative Lax norm $\sup \left\{|(v, w)| /\|w\|_{s}: w \in E_{\infty} \backslash\{0\}\right\}$, where the inner product in $H$ and the duality relation introduced by it will be denoted by the same symbol. We denote the inner product (and also the corresponding duality rela= tion) of the Hilbert space $H^{s}$ by $(\cdot, \cdot)_{s}$ and note that, for $a \geqq 0, v \in H^{s+a}$ and $\dot{w} \in H^{s-a}$, we have $\left|(v, w)_{s}\right| \leqq\left\|v_{s+a}\right\| w \|_{s-u}$.

Lemma 2.1: Suppose that $-\infty<k<l<\infty$. Then the imbedding $H^{l} \subset H^{k}$ is - continuous, and compact:

Proof: Let $\left\{v^{\mu}\right\}$ be a sequence converging to $v$ weakly in $H^{\prime},\left(v^{\mu}-v, w\right) \rightarrow 0$ as $\mu \rightarrow \infty$ for all $w \in E_{\infty}$. Using the Fourier expansion of $v^{\mu}$ and $v$ in the orthonormal basis $\left\{e_{i}\right\}$ we get $\left(v_{i}{ }^{\mu}-v_{i}\right) \rightarrow 0$ as $\mu \rightarrow \infty$ for all $i$, where $v_{i}{ }^{\mu}, v_{i}$ are the Fourier coefficients of $i^{\mu}$ and $v$, respectively. For every fixed $i^{*}$ and any $\varepsilon^{\prime}>0$ we find a $\mu_{0}$ $=\mu_{0}\left(i^{*}, \varepsilon\right)>0$ such that

$$
\sum_{i=1}^{i *} \dot{\lambda}_{i}^{k}\left|\hat{v}_{i}{ }^{\mu}-v_{i}\right|^{2} \leqq \varepsilon / 2 \quad \text { for all } \mu \geqq \mu_{0}
$$

Since the sequence $\left\{v^{\mu}\right\}$ is boúnded in $H^{l}$ and $\lambda_{i} \rightarrow \infty$, there exists an $i^{*}=i^{*}(\varepsilon)$, which does not'depend on $\mu$, such that

$$
\sum_{i=i^{*}+1}^{\infty} \lambda_{i}{ }^{k}\left|v_{i}{ }^{\mu}-v_{i}\right|^{2} \leqq \lambda_{i^{*}}^{k-l} \sum_{i=1}^{\infty} \lambda_{i}{ }^{l}\left|v_{i}{ }^{\mu}-v_{i}\right|^{2} \leqq \varepsilon / 2
$$

Combining both inequalities we get $\left\|v^{\mu}-v\right\|_{k}<\varepsilon$ for all $\mu>\mu_{0}$, which means $v^{\mu} \rightarrow v$ strongly in $H^{k}$.

Similarly to the second inequality one can show that for all $v \in H^{k}$. the inequality . $\|v\|_{k} \leqq \lambda_{1}{ }^{(k-l) / 2}\|v\|_{l}$ holds, from which the continuity of the imbedcling follows

Lem ma 2.2: Suppose that $-\infty<k \leqq l \leqq m<\infty$ with $k \leqslant m$. Then, for $v \in H^{\prime}$, the interpolation inequality $\|v\|_{l} \leqq\|v\|_{k}^{(m-l) /(m-k)}\|v\|_{m}{ }^{(l-k) /(m-k)}$ holds.

今े roof: Applying the Hölder inequality with the exponents $p=(m-k) /(m-l)$, $q=(m-k) /(l-k)$ to the definition of the norm $\|v\|_{l}$ we get the assertion

We will denote the Banach space of all measurable and $L_{p}$-integrable vector functions $y(\cdot):[0, T] \rightarrow H^{k}$ by $L_{p}\left(0, T ; H^{k}\right)$ with the norms

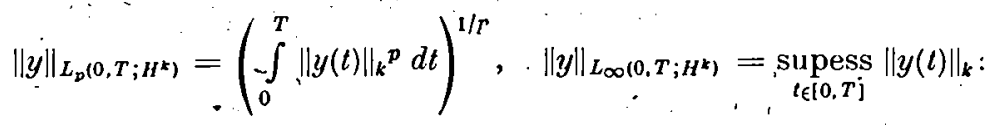


Let $Y_{p . q}^{k, l}=\left\{y \in L_{p}\left(0, T ; H^{k}\right): \dot{y} \in L_{q}\left(0, T ; H^{i}\right)\right\}$ be the. Banach space with the norm $\|y\|_{Y_{p, q}^{k, i}}=\|y\|_{L_{p}\left(0, T ; H^{k}\right)}+\|\gamma\|_{L_{q}\left(0, T ; H^{l}\right)}$.

Lemma 2.3: Suppose that $\dot{y} \in Y_{p, q}^{k . l}$ with $l<k, 1 \leqq p<\infty, 1<q<\infty$. Thei $y \in L_{\infty}\left(\tilde{0}, T ; H^{m}\right)$ with

$$
m \leqq M(k, l, p, q)=\left\{\begin{array}{cc}
\frac{k p(q-1)+l q}{p(q-1)+q} \text { if } 1 / p+1 / q \geqq 1 \\
(k+l) / 2- & \text { if } 1 / p+1 / q \leqq 1,
\end{array}\right.
$$

and, for every $\varepsilon>0$ and $x$ with $1 \geqq x>K(p, q):=\max \{1 / 2, q /(p(q-1)+q)\}$, there exist constants $c_{1}(\dot{\varepsilon}), c_{2}(\varkappa)$ with $c_{2}(1)=1$, and $c_{3}>0$ such that, for all $y \in Y_{p, q}^{k, l}$,

$$
\sup _{t \in[0, T]}\|y(t)\|_{m} \leqq \varepsilon\|\dot{y}\|_{L_{e}(0, T ; H)}^{x^{\prime}}+\dot{c}_{1}(\varepsilon)\|\dot{y}\|_{L_{p}\left(0, T ; H^{k}\right)}^{c_{1}(x)}+c_{3}\|y\|_{L_{p}\left(0, T ; H^{k}\right)} .
$$

Proof: By virtue of Lemma 2.1 we only have to prove the lemma for $m=M(k$, $l, p, q)$. It follows from $d(y(t), y(t))_{m} / d t=2(\dot{y}(t), y(t)\rangle_{m}$ that, for all $t \in[0, T]$ and almost all $t_{0} \in[0, T]$, we have

$$
\begin{aligned}
\|y(\ell)\|_{m}^{2} & =\left\|y\left(t_{0}\right)\right\|_{m}^{2}+2 \int_{t_{0}}^{t}(\dot{y}(\tau), y(\tau))_{m} d \tau \\
\because & \leqq\left\|y\left(t_{0}\right)\right\|_{m}^{2}+2 \int_{0}^{T}\|\dot{y}(\tau)\|_{l}\|y(\tau)\|_{2 m-l} d \tau \\
& \leqq\left\|y\left(t_{0}\right)\right\|_{m}^{2}+2\|\dot{y}\|_{L_{q}\left(0, T ; H^{l}\right)}\left(\int_{0}^{T}\|y(\tau)\|_{2 m-l}^{q /(q-1)} d \tau\right)
\end{aligned}
$$

By definition of $m$ we have $m \leqq 2 m-l \leqq k$. So we can use the interpolation iniequality from Lemma 2.2 in the form

$$
\|y(\tau)\|_{2 m-l} \leqq\|y(\tau)\|_{m}{ }^{(k+l-2 m) /(k-m)}\|y(\tau)\|_{k^{(m-l) / k-m)}}
$$

to estimate the last integral. Since $q(m-l) /((k-m)(q-1)) \leqq p$ we get

$$
\|y(t)\|_{m}^{2} \leqq\left\|y\left(t_{0}\right)\right\|_{m}^{2}+c\|\dot{y}\|_{L_{d}\left(0, T ; H^{\prime}\right)}\|y\|_{L_{\infty}\left(0 . T^{\prime} ; H^{m}\right)}^{\frac{k+l-2 m}{k-m}}\|y\|_{L_{p}\left(0, T ; H^{k}\right)}^{\frac{m-l}{k-m}}
$$

In the case $m<(k+l) / 2$, it follows from Young's inequality that, for $\varkappa_{s}>(k-m)$ / $(k-l)=K(p, q)$,

$$
\begin{aligned}
& \|\ddot{y}(t)\|_{m}^{2} \leqq\left\|y\left(t_{0}\right)\right\|_{m}^{2}+\frac{1}{2}\|y\|_{L_{\infty}\left(0, T ; H^{m}\right)}^{2}+\frac{\varepsilon^{2}}{2}\left\|\dot{y}_{\|}\right\|_{L_{q}\left(0, T ; H^{\prime}\right)}^{2 \times}
\end{aligned}
$$

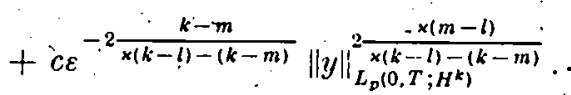

Here and later we will denote different constants with the same symbol $c:$ We can choose $t_{0}$ such that $\left\|y\left(t_{0}\right)\right\|_{m} \leqq T^{1 / p}\|y\|_{L_{p}\left(0, T ; H^{k}\right)}$. Taking now the supremum with respect to $t \in[0, T]$ in the last inequality we get the assertion of our lemma. In the' case $m=(k+l) / 2$ the same is true for $x>1 / 2$

We will denote the operator restricting a function $y(\cdot)$ to $t=\tau$ by $\gamma_{t}: \gamma_{\mathrm{r}} y^{\prime}=y(\tau)$. From the previous lemma it follows, for $m \leqq M(k, l, p, q)$, that the operator $\gamma_{t}$ : $Y_{p, q}^{k, l} \rightarrow H^{m}$ is uniformly continuous with respect to $t \in\left[0, T^{\prime}\right]$. 
Lemma 2.4: Suppose that $i<k^{*}<k, 1<p<\infty, 1 \leqq q<\infty$. Then the imbedding $Y_{p, q}^{k, l} \subset L_{p}\left(0, T ; H^{k^{*}}\right)$ is compact.

- Proof: For $q>1$ the lemma is proved in [7: Theorem 4.4.1] and for $q=1$ the proof given in [7: Theorem 7.4.1] can be extended from $p=2$ to arbitrary $p \in$ (1, $\infty)$

Lemma 2.5: Suppose that $1 \leqq p<\infty,-\infty<l \leqq k-2$. Then for all $q$ with $\alpha(l) \leqq 1 / q<1$ the operator $A: Y_{p, q}^{k, l} \rightarrow L_{q}\left(0, T ; H^{l}\right)$ is bounded, and, for every $\dot{\varepsilon}>0$, there exists a constant $c_{A}(\varepsilon)$ not depending on $y$ such that

$$
\text { ' }\|A y\|_{L_{q}\left(0, T ; H^{i}\right)} \leqq \varepsilon\|\dot{y}\|_{L_{q}\left(0, T ; H^{\imath}\right)}+c_{A}(\varepsilon)\|y\| \|_{L_{p}\left(0, T ; H^{k}\right)}, \cdots
$$

where $a(l)$ is given by

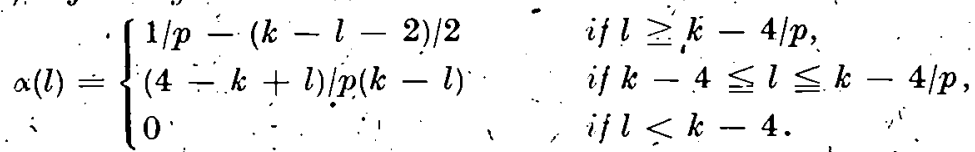

Proof: Let $m^{*} \leqq l+2$ be a real number and $y \in Y_{p, q}^{k . l}$. Then we use the interpolation inequality from Lemma 2.2, Young's inequality and (2.1) with $x=1$ to estimate $A y$ :

$$
\begin{aligned}
& \|A \dot{y}\|_{L_{0}\left(0 . T ; H^{i}\right)}^{q}=\|y\|_{L_{q}\left(0 . T ; H^{1+2}\right)}^{q}=\int_{0}^{r} \|\left. y(t)\right|_{l} ^{q} d,
\end{aligned}
$$

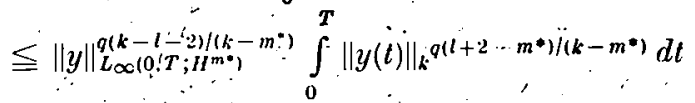

$$
\begin{aligned}
& \leqq_{1}\left(\varepsilon . \mid \dot{y}\left\|_{L_{L_{q}\left(0, T ; H^{l}\right)}}+c_{A}(\varepsilon)\right\| \dot{y} \|_{L_{p}\left(0, T ; H^{k}\right)}\right)^{q} .
\end{aligned}
$$

The right-hand -side is bounded, provided the eonditions $m^{*} \leqq M(k, l, p, q)$ (cf. Lemma 2.3) and $p \geqq q\left(l+2-m^{*}\right) /\left(k<m^{*}\right)$ are satisfied. Since this is clear for $p \geqq q$ we only consider the case $p<q$. We have to ensure the existence of a number $m^{*}$. with $\left(q\left(l_{0}+2\right)-p k\right) /(q-p) \leqq m^{*} \leqq M(k, l, p, q)$. However, this is clone by the choice of $q$ given in the lemma

Now we introduce a continuous nonlinear operator

$$
B: H^{m} \rightarrow H^{n} \text { with }\|B(y)\|_{n} \leqq c\left(\|y\|_{m}{ }^{\top}+1\right), r>1 \text {. }
$$

Lemmà 2.6: Suppose that $1 \leqq p<\infty, 1<r<p+1, m \leqq k$ and $l_{0}<l<\infty$ with

$$
l_{0}= \begin{cases}-\infty & \text { if } p \geqq r, \\ (r m-p k) /(r-p) & \text { if } r-1<p<r .\end{cases}
$$

Then for all $q$ with $\beta(l) \leqq 1 / q<1$ the operator $B: Y_{p, q}^{k, l} \rightarrow L_{q}\left(0, T ; H^{n}\right)$ is bounded and continuous, and, for any $\dot{\varepsilon}>0$, there exist constants $c_{B}(\varepsilon)>0, c_{B}>r$ not depending on'y such that

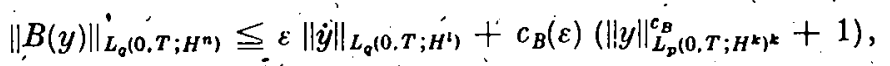

where $\beta(l)$ is given by

$$
\beta(l)= \begin{cases}\frac{r}{p} \frac{p(k-m)-(m-l)}{r(k-m)-(k-l)} \text { if } l_{0}<l \leqq l_{1}, \\ \frac{r}{p} \frac{2 m-k-l}{k-l}, & \text { if } l_{1}<l<k-r(k-m) \\ \frac{r-2}{p} \cdot \delta, & \text { if } k-r(k-m) \leqq l\end{cases}
$$



with $\dot{\delta}>0, l_{1}=-\infty$
$r-1<p<r+1$ for $p \geqq r+1$ and $l_{1}=\bar{m}-\frac{r+p-1}{r-p+1}(k-m)$ for

Proof: For $y \in Y_{p, q}^{k, t}$ we haye

$$
\|B(y)\|_{L_{q}\left(0 . T ; H^{n}\right)}^{q}=\int_{0}^{r}\|B(y(t))\|_{n^{q}} d t \leqq c_{1}+c_{2} \cdot \int_{0}^{T}\|y(t)\|_{m}^{r q} d t
$$

If $r q \leqq p$, the boundedness of $B$ and the validity of $(2.4)$ are obvious since $m \leqq k$. We suppose now $r q>p$ and use the interpolation inequality from Lemma 2.2 in the form

$$
\|y(t)\|_{m} \leqq\|y(t)\|_{m^{*}}^{(k-m) /\left(k-m^{*}\right)}\|y(l)\|_{k}^{\left(m-m^{*}\right) /\left(k-m^{*}\right)}, \quad m^{*} \leqq m \leqq k,
$$

where $m^{*} \leqq M(k, l, p, q)$ (cf. Lemmía 2,3 ). Then we get from Lenıma 2.3

$$
\begin{aligned}
& \|B(y)\|_{L_{e}\left(0, T ; H^{n}\right)}^{q^{v}} \leqq c_{1}+c_{2}\|y\|_{L_{\infty}\left(0 . T ; H^{m}\right)^{*}}^{r-\frac{k-m}{k-m^{*}}} \int_{0}^{T}\|y(t)\|_{k}^{r q \frac{m-m^{*}}{k-m^{*}}} d t \\
& \leqq\left(\varepsilon\|\dot{y}\|_{L_{0}\left(0, T_{;} ; H^{*}\right)}^{\alpha r \frac{k-m}{k-m^{*}}}+c_{B}(\varepsilon)\left(\|y\|_{L_{p}\left(0, T ; H^{*}\right)}^{c_{B}}+1\right)\right)^{\phi}
\end{aligned}
$$

if the conditions $x>K(p, q)$ and $r q \frac{m-m^{*}}{k-m^{*}} \leqq p$ are, satisfied. Additionally, we chose $x$ sich that $x \dot{r} \frac{k-m}{\not k-m^{*}}=1$. All these conditions are satisfied if there exists a. number $m^{*} \leqq M(k, l, p, q)$ with $\frac{r q m-p k}{r q-p} \leqq m^{*}=k-x r(k-m)<\dot{k}$ - $K(p, q) r(k-m)$. Noting that $M(k, l, q, p)<k-K(p, q) r(k-m)$ holds for all $l<k-r(k-m)$, we derive the existence of such a number $m^{*}$ by easy cal. culations from the assumptions of the lemma formulated for $l$ and $1 / q$. The continuity of the operator follows from the continuity of $B: H^{m} \rightarrow H^{n}$, from the proved boundedness and from Lebesgue's dominated convergence theorem

\section{The control problem.}

Let $Y_{1}, Y$ and $\dot{U}$ be reflexive Banach spaces with $Y_{1} \subset Y$. Let $U_{\text {ad }}$ be a convex closed subset of $U$, and let: $J: Y \times U^{\prime} \rightarrow \mathbb{R}$ be a convex funetional. From [1] we will quote an existence theorem for general extremal problems of the type

$$
\begin{aligned}
& J(y, u) \rightarrow \inf , \\
& L(y, u)+F(y)=0, \quad u \in U_{\mathrm{alt}},
\end{aligned}
$$

where the operators $L: Y_{1} \dot{\times} U \rightarrow W$ and $F: Y_{1} \rightarrow W$ act in a Banach space $\dot{W}$.

A pair $(y, u) \in Y_{1} \times U_{\text {ad }}$ which satisfies (3.2) and for $\Varangle$ which $J(y, u)<\infty$ is called an admissible element of the problem $(3,1),(3.2)$. We denote the set of admissible elements of (3.1), (3.2) by $\Phi^{\prime}$. A pair $\left(y^{*}, u^{*}\right) \in \mathbb{Q}$ is called a solution of (3.1), (3.2) if it satisfies $J\left(y^{*}, u^{*}\right)=\inf _{\mathbb{Q}} J(y, u)$.

Theorem 3.1: Suppose that the following assumptions hold true:

i) the convex functional $J: Y \times U \rightarrow \mathbb{R}$ is bounded below and lower semicontinuous,

ii) the imbedding $Y_{1} \subset Y$. is continuous, 
iii) the operator $L: Y_{1} \times U \rightarrow W$ is linear and continuoùs,

iv) the operator $F: Y_{1} \rightarrow W$ is wéakly sequentially continuous,

v) the set $\mathbb{Q}$ is non-emply,

vi) for all $R>0$ the set $\{(y, u) \in \mathbb{Q}: J(y, u) \leqq R\}$ is bounded in $Y_{1} \times U$.

Then the problem (3.1.), (3.2) has a solution $\left(y^{*}, u^{*}\right) \in Y_{1} \times U_{\mathrm{ad}}$ :

Proof: The proof proceeds exact]y as in [1: Theorem 5.1]. The only difference is that we dò not need the space $Y_{-1}$ which was compactly inibedded in $Y_{i 1}$. The assumption in [1] concerning the demicontinuity of the operator $F: Y_{-1} \rightarrow W$ here is replaced by our assumption iv). From this it follows directly that, for a subsequence $\left\{y_{\mu}\right\}$ of a minimizing sequence with $y_{\mu} \rightarrow y$ weakly in $Y_{1}$; also $\left\langle F\left(y_{\mu}\right), v\right\rangle_{W} \rightarrow\langle F(y), v\rangle_{W}$, for all $v \in S$, where $S$ is a dense subset of $W^{*}$

Remark 3.1: Theorem 3.1 is in accordance with the existence theorem for general extremal problems in [3]. With the help of our assumptions concerning the function spaces and operators we can ensure that the conditions of compactness and closedness formulated in [3: Theoren 3.1.11] are satisfied for the,problem (3.1), (3.2).

\section{Results}

U'sing Theorem' 3.1 weprove in this section an existence theorem for control problems - of general nonlinear evolution equations. To this purpose we specify the function spaces, operiators and the functional $J$ in Theoren 3.1 as follows. For real numbers $k, l, l^{*}, p, q, q^{*}$ with $1<p, q, q^{*}<\infty^{\prime}$ we set $Y=L_{p}\left(0, T^{*} ; H^{k}\right), \quad Y_{1}=Y_{p, q^{*}}^{k, l^{*}}, U$ $=L_{q}\left(0, T ; H^{\prime}\right)$ and $W=L_{q^{*}}\left(0, T ; H^{l^{*}}\right) \times H^{l^{*}}$. With the operators $A: H^{k} \rightarrow H^{k-2^{\prime}}$ and $B: H^{m} \rightarrow H^{n}$ defined in Section' 2 (cf. (2.3)) we set, for $v \in \mathbb{R}$,

$$
L(\dot{y}, u)=\left(\dot{y}+v A y-u, \gamma_{0} y\right), \quad F(\dot{y})=\left(B(y),-y_{0}\right) .
$$

With a convex functional $\Phi: H^{k} \times H^{l} \rightarrow \mathbb{R}$ satisfying the conditions

$$
\begin{aligned}
& \sup _{\|y\|_{k}+\|u\|_{l} \leqq R} \Phi(y, u)<\infty \quad \text { for all } R>0 \\
& \Phi(y, u) \geqq c_{1}\left(\|y\|_{k}^{p}+\|u\|_{l}^{q}\right)-\dot{c}_{2} \quad \text { with } c_{1}, c_{2}>0
\end{aligned}
$$

we set $J(y, u)=\int_{0}^{T} \Phi(y(t), u(t)) d t$. Now we. can write the problem (3.1), (3.2) for $(y, u) \in Y_{1} \times U_{\text {ad }}$ in the form

$$
\begin{aligned}
& J(y, u)=\int_{0}^{T} \Phi(y(t), u(t)) d ! \rightarrow \text { inf } \\
& \dot{y}+\nu A y+B(y)=u, \ldots \text { with } \gamma_{0} y=y_{0}
\end{aligned}
$$

where $U_{\mathrm{ad}}$ is a convex closed subset of $U$ and $y_{0} \in H^{\circ}, v \in \mathbb{R}$.

Theorem 4.1: Suppose that $v \in \mathbb{R}, \dot{n}<k, 1<r<p+1$ and that the set $\mathbb{Q}$ of admissible elements of (4.3), (4.4) is non-empty. Then, for any $l \in \mathbb{R}, 1<q<\infty$, there exists a solution $\left(y^{*}, u^{*}\right) \in Y_{p . q^{*}}^{k . l^{*}} \times U_{\text {ad }}$ of $(4.3),(4.4)$ if $l_{0}<l^{*} \leqq \min (l, n, k-2)$ and $1>1 / q^{*}>\max \left(1 / q, \alpha\left(l^{*}\right) ; \beta\left(l^{*}\right)\right)$, where $l_{0}, \alpha(\cdot), \beta(\cdot)$ are defined in Lemma 2.5 and Lemma 2.6 .

Proof: For $q^{*}>1$ all of the spaces $Y_{1}, Y, U$ are reflexive Banach spaces. We prove that all of the assumptions $i$ ) - vi.) of Theorem 3.1 hold true. 
i) Since $\Phi$ is convex the, functional $J$ in (4.3) is convex and because of (4.2) it is bounded below. The fact that $J$ is lower semicontinuous is proved in [ 1 : Theorem 6.1 ]

ii) The continuity of the imbedding $Y_{1} \subset Y$ follows from Lemma 2.4.

iii) The continuity of the operator $L: Y_{1} \times U \rightarrow W$ defined in (4.1) follows from the inequalities

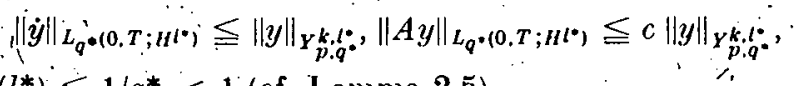

where $\alpha\left(l^{*}\right) \leqq 1 / q^{*}<1$ (cf. Lemma 2.5),

and

$$
\|u\|_{L_{q} \cdot\left(0, T ; H^{*}\right)} \leqq\|u\|_{L_{q\left(0, T ; H^{l}\right)},} \quad \text { for } l^{*} \leqq l, q^{*} \leqq q
$$

(cf.'Lemma 2.3).

$$
\left\|\gamma_{0} y\right\|_{H^{*}} \leqq c \mid ! y \|_{\gamma_{p, q^{*}}^{k, l^{*}}}, \quad \therefore \text { since } l^{*} \leqq M\left(\dot{k}, l^{*}, \ddot{p}, q^{*}\right)
$$

iv) We prove that the nonlinear operator $F: Y_{1}^{\prime} \rightarrow^{\prime} W$ defined in (4.1), is weakly sequentially continuous. To this purpose let $\left\{y_{\mu}\right\} \subset Y_{1}$ be a sequence with $y_{\mu} \rightarrow y$ weakly in $\dot{Y}_{1}$. We show that, for any element $v$ of the dense subset $S=C\left([0 ; T] ; E_{\infty}\right)$

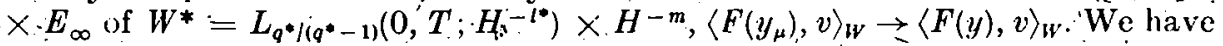

$$
\begin{aligned}
& \left\langle F\left(y_{\mu}\right)-F(y), v\right\rangle_{W}=\int_{0}^{T}\left(B\left(y_{\mu}(t)\right)-B(y(t)), v(t)\right) d t \\
& \leqq\|v\|_{C\left(\{0, T\} ; H^{-n}\right)} \int_{0}^{T}\left\|B\left(y_{\mu}(t)\right)-B(y(t))\right\|_{n} d t .
\end{aligned}
$$

By virtue of Lemma 2.4, for $m \leqq k^{*}<k$, the imbedding $Y_{p, q^{*}}^{k, \cdot} \subset L_{p}\left(0 ; T^{\prime} ; H^{k^{*}}\right)$ is compact. That is why, $y_{\mu} \rightarrow y$ strongly in $L_{p}\left(0, T ; H^{k^{*}}\right)$, and therefore, for almost all $t \dot{\epsilon}[0, T] ;\left\|y_{\mu}(t)-y(t)\right\|_{m} \leqq c\left\|y_{\mu}(t)-y(t)\right\|_{k^{*}} \rightarrow 0$ as $\mu \rightarrow \infty$. Since $B: \dot{H}^{m} \rightarrow H^{n}$ is a. continuous operator the convergence of the integral in (4.5) follows from the esti-

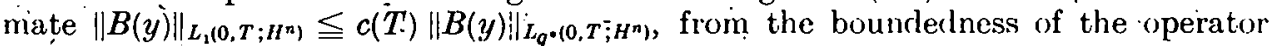
$B: Y_{1} \rightarrow L_{q^{\bullet}}\left(0, T^{\prime} ; H^{n}\right)$ proved in Lemma 2.6 and from the boundedness of the sequence. $\left\{y_{\mu}\right\}$ in $Y_{1}$.

v) $\mathbb{Q} \neq \varnothing$ is a hypothesis of our theorem.

vi) As a consequence of the second inequality, of (4.2) we get, for an admissible pair $(y, u)$ of $(4.3),(4.4)$, from $J(y, u) \leqq R$ that, $\|y\|_{L_{p}\left(0, T ; H^{k}\right)}+\|u\|_{L_{q}\left(0 . T ; H^{\natural}\right)} \leqq C(R)$. Using the estimates (2.2), (2.4) with $\varepsilon=1 / 4|v|$, (if $\nu \neq 0$ ), $\varepsilon=1 / 4 c$, respectively, we conclude

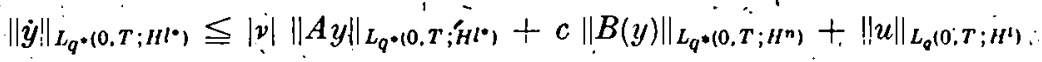

$$
\begin{aligned}
& \leqq 1 / 2\left|\dot{y}\left\|_{L_{q^{*}\left(0, T ; H l^{*}\right)}}+C\left(1_{0}^{*}+\|y\|_{L_{p}\left(0, T ; H^{k}\right)}^{c_{\dot{B}}}\right)+\right\| \dot{u}\right|_{L_{q}\left(0 . T ; H^{\natural}\right)}
\end{aligned}
$$

From this estimate it follows that the set $\{(y, c) \in \mathbb{Q}: J(y, u)<R\}$ is bounded in $\dot{Y}_{\mathrm{i}} \times U$

Remark 4.1: Theorem 4.1 is a generalization of [1: Theorem 6.1].. There the state equation of the control problem was the Navier; Stokes system with arbitrary viscosity $v \in \mathbb{R}$ in a bounded domain $\Omega \subset \mathbb{R}^{N}, N=2,3 ; \ldots$, with' $\partial \Omega \in C^{\infty}$. The nonlinear operator $B$ in the corresponding operator equation was characterized by the estimate (2.3) with $n<-(N / 2+1), m=0$ and $r \stackrel{\prime}{=} 2$. Under the same assumptions concerning the objective functional as in [1: Theorem 4.1$]$ the existence of an optimal pair of state and control $\left(y^{*}, u^{*}\right) \in Y_{p, q^{*}}^{k . l^{*}} \times L_{q}\left(0, T ; H^{l}\right)$ with $k>0$, $p \geqq 2, l^{*}<\min (-(N / 2+1), l)$ and $1 / q^{*}=\max (1 / q, 2 / p)$ was proved. As a consequence of our Theorem 4.1 the region of parameter values $p ; q^{*}$ for which a solution. $y^{*} \in Y_{p, a^{*}}^{k, q^{*}}$ exists is entarged. The values of $p$ and $q^{*}$ can be chiosen as functions of $k>m=0, r \stackrel{p, q^{*}}{=} 2 \cdot$ and $l^{*}$, $<\min (-(N / 2+1), l, k-2)$ as follows: $p>-2 l^{*} /\left(k-l^{*}\right)$ and $1 / q^{*}>\max \left(1 / q, \alpha\left(l^{*}\right), \beta\left(l^{*}\right)\right)$. This means that the case $p=2$ investigated in [1: Theorem 6.6] does not need special considerations. Moreover, Theorem 4.1 yields $y^{*} \in Y_{p, q^{*}}^{k, l^{*}}$ also for $p<2$ and $q^{*}>1$. 


\section{REFERENĆES}

[1] Фурсиков, А.' В.: Задачи управления и.теоремы, касаюциеся однозначной разрешимости смешанной краевой задачи для трехмерных уравиени Н Навье-Стокса и Эйлера. Мат. сб. 115 .(157) (1981), $281-307$.

[2] Фурсйков, А. В.: Свойтва решенйй экстремальных задач связаниых с системой Навье-Стокса. Ма́т. сб. 118 (160) (1982), 3.23-349. а

[3] KLUge, R.: . Nichtlineare Variationsungleichungen und Extremalaufgaben. Berlin: Dt. Verlag Wiss. 1979.

[4] LANGMACH, H.: On the determination of functional parameters in some parabolic differential equations. In: Theory of nonlinear operators (Abh. Akad. Wiss. DDR: Bd. 6 ; ed.: R. Kluge). Berlin: Akademie-Verlag 1978, p. 175- 184.

[5] Lrons, J. L.: Contrôlè des systèmes.distribués singuliers.' Paris: Gauthier-Villars 1983.

[6] NÜrNBERG, R.: On the determination of functional para meters̀ in nonlinear evolution equations of Nivier-Stokes type. In: Inverse and improperly posed problems in differential equations (Mathem.' Research: Vol. 1;- ed: G. Anger). Berlin: Akademie-Verlag 1979, p. $189-196$.

[7] VIŠrK, M. I., und A. V. Fursukov:- Mathematische Probleme der statiștischen Hydromechanik. Leipzig: Akad. Verlagsges. Geest \& Portig 1986.

Manuskripteingang: 16. 03. 1988; in revidierter Fassung 25.11.1988

\section{VERFASSER}

D'́r. Bernd Krause

Sektion Mathematik/Naturwissenschaften/Informatik der Ingenieurhochschule

Bernburger Str..52-57

DDR- 4370 Köthen 